\title{
Asymptomatic Duodenal Metastasis From Small Cell Lung Cancer Diagnosed by Endoscopic Ultrasound-Guided Fine- Needle Aspiration
}

\author{
Akira Imoto ${ }^{\mathrm{a}, \mathrm{d}}$, Daisuke Masuda ${ }^{\mathrm{a}}$, Atsushi Okuda ${ }^{\mathrm{a}}$, Wataru Takagi ${ }^{\mathrm{a}}$, Saori Onda $^{\mathrm{a}}$, Tatsushi Sano ${ }^{\mathrm{a}}$, \\ Mio Amano ${ }^{a}$, Usama Abderaala, b, Takeshi Ogura ${ }^{a}$, Yoshitaka Kurisuc ${ }^{c}$, Kazuhide Higuchi ${ }^{a}$
}

\begin{abstract}
A 67-year-old man with small cell lung cancer was referred to our department. The patient had received chemotherapy for lung cancer and complained of no specific symptoms. Follow-up positron emission tomography (PET) showed a new duodenal lesion. Immunohistochemistry (IHC) with the specimens obtained by endoscopic ultrasound-guided fine-needle aspiration (EUS-FNA) showed that the lesion was metastasis from lung cancer. These results contributed to the subsequent treatment strategy. Gastrointestinal metastasis from lung cancer diagnosed during the patient's lifetime is rare. This is the first case of asymptomatic duodenal metastasis from small cell lung cancer diagnosed by EUS-FNA.
\end{abstract}

Keywords: Gastrointestinal metastasis; Small intestine; Small cell lung cancer; Endoscopic ultrasound-guided fine-needle aspiration

\section{Introduction}

Lung cancer is a malignant tumor with high incidence and mortality. Approximately one-half of patients with lung cancer have metastatic disease at the time of initial diagnosis. Distant metastases are usually found in the lymph nodes, lungs, liver, adrenal glands, brain, and bone [1,2]. Small intestinal metastases from lung cancer are rare, reportedly in $2-11 \%$ of autopsy cases, and cases that are clinically apparent during the patient's lifetime are even less common [3]. Endoscopic ultrasound-guided fine-needle aspiration (EUS-FNA) is an established method of obtaining submucosal tissue specimens [4]. The case of a patient with duodenal metastasis from small cell

\footnotetext{
Manuscript accepted for publication August 18, 2016

aSecond Department of Internal Medicine, Osaka Medical College, Takatsuki, Osaka, Japan

bepartment of Gastroenterology, Sohag University, Sohag, Egypt 'Department of Pathology, Osaka Medical College, Takatsuki, Osaka, Japan ${ }^{\mathrm{d} C}$ Corresponding Author: Akira Imoto, Second Department of Internal Medicine, Osaka Medical College, 2-7 Daigaku-machi, Takatsuki-shi, Osaka 5698686, Japan. Email: akiraimoto27@gmail.com
}

doi: http://dx.doi.org/10.14740/jmc2624w lung cancer diagnosed by EUS-FNA is described.

\section{Case Report}

A 67-year-old man with a history of hypertension was referred to our hospital because of a 60 -mm mass shadow in the right lung hilar region (Fig. 1). He smoked 30 cigarettes daily for 40 years. The histological diagnosis was small cell lung cancer (limited disease, cT2bN2M0 stage IIIA) [5] by immunohistochemical (IHC) examination of specimens obtained by bronchoscopy, and he was treated by chemoradiotherapy. Cisplatin and etoposide were administered as first-line chemotherapy with radiation, but therapy was stopped after five courses because of radiation pneumonitis. Irinotecan was used as secondline, but it was also stopped, due to diarrhea, after two courses. Because new lesions appeared in the left lobe of the lung and lumbar vertebrae 3 months after second-line chemotherapy was stopped, third-line amrubicin was started. Just before the 10th cycle of amrubicin, follow-up positron emission tomography

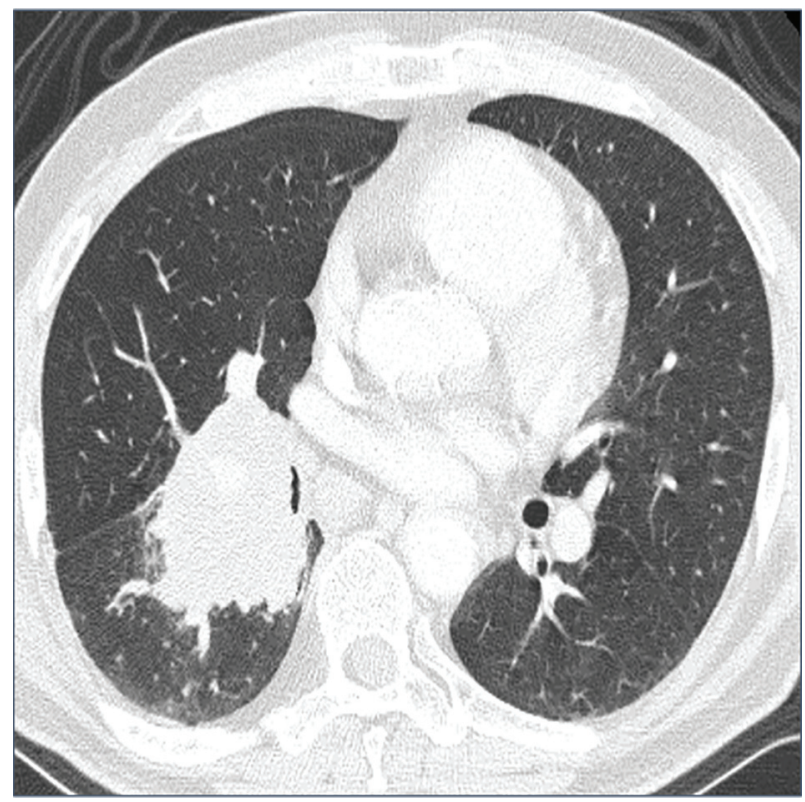

Figure 1. Computed tomography (CT) shows a $60-\mathrm{mm}$ mass shadow in the right lung hilar region. 


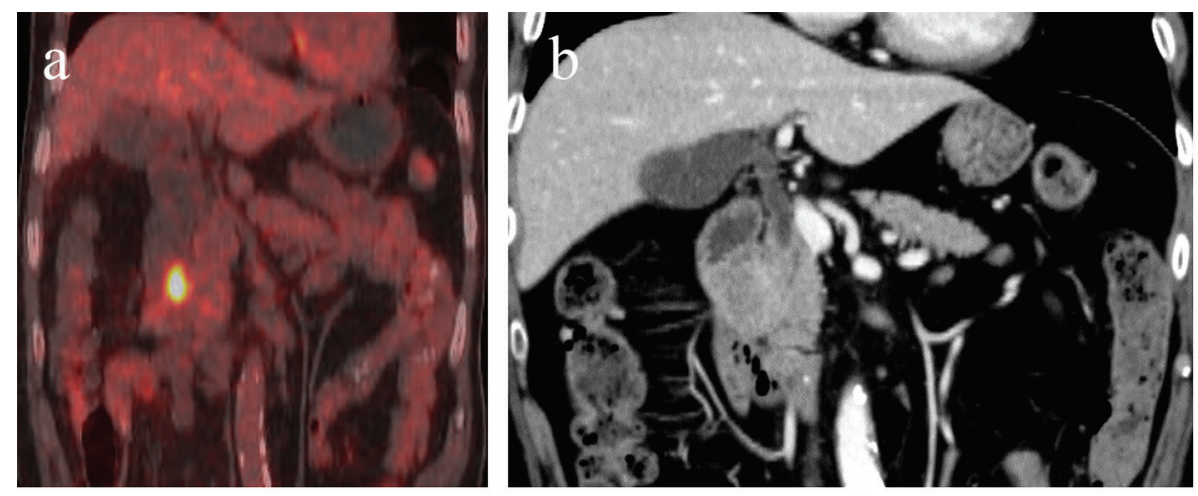

Figure 2. Positron emission tomography (PET) shows accumulation of fluorodeoxyglucose (FDG) in the duodenal descending limb (a). Mildly enhanced mass was detected in the same position on contrast-enhanced computed tomography (CT) (b).

(PET) showed accumulation of fluorodeoxyglucose (FDG) in the duodenal descending limb (Fig. 2a). Mass with mild enhancement was detected at the position on contrast-enhanced computed tomography (CT) (Fig. 2b). The patient was referred to our department for diagnosis of the duodenal lesion.

On examination, his temperature was $36.9^{\circ} \mathrm{C}$, heart rate was 80 beats per minute, blood pressure was 109/49 mm Hg, and respiratory rate was 16 breaths per minute. Laboratory tests showed a mild inflammatory response with no biochemical abnormalities. The levels of carcinoembryonic antigen (CEA), neuron-specific enolase (NSE), squamous cell carcinoma (SCC) antigen, and sialyl Lewis X-i (SLX) antigen were not elevated, but cytokeratin 19 fragment (CYFRA) 21-1 and pro-gastrin-releasing peptide (ProGRP) were increased (5.0 $\mathrm{ng} / \mathrm{mL}$ and $166 \mathrm{pg} / \mathrm{mL}$, respectively).

On upper gastrointestinal endoscopic examination, a huge submucosal tumor (SMT) was found in the duodenal descending limb (Fig. 3), which was thought to be the lesion detected on PET. The SMT was visualized as $30 \mathrm{~mm}$ in size, roundshaped, homogeneous, and low echoic mass connected with fourth layer of duodenal wall on radial type EUS (Fig. 4a). The

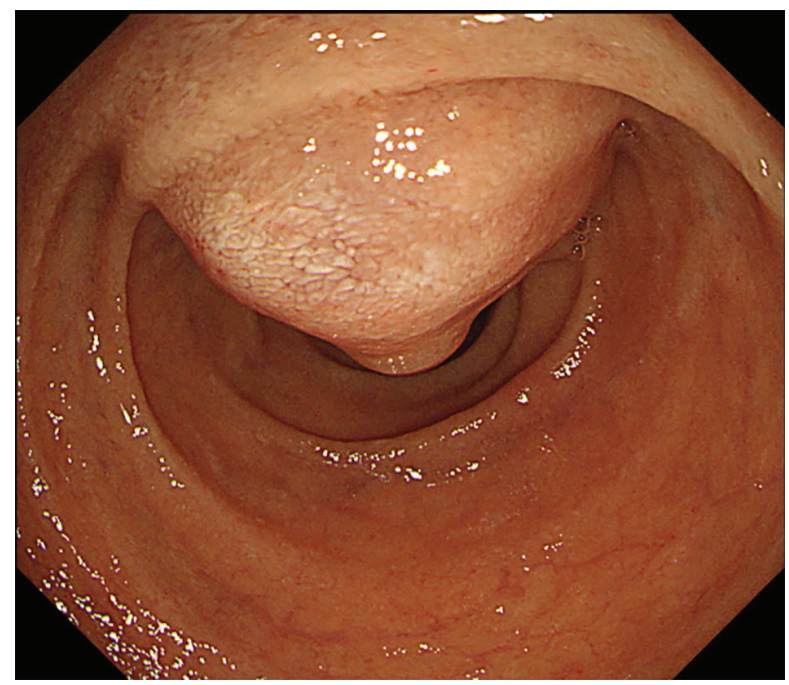

Figure 3. Upper gastrointestinal endoscopic examination shows a huge submucosal tumor (SMT) in the duodenal descending limb. first impression based on the examination was gastrointestinal stromal tumor (GIST) of the duodenum. Hematoxylin and eosin (HE)-stained sections of specimens obtained by EUS-FNA (Fig. 4b) showed relatively small atypical cells with a high nuclear/cytoplasm ratio (Fig. 5a), indicating a metastasis from primary lung cancer (Fig. 5b). This diagnosis was confirmed by IHC examination; the tumor cells were diffusely positive for cluster of differentiation 56 (CD56) (Fig. 6a) and thyroid transcription factor 1 (TTF-1) (Fig. 6b) and focally positive for chromogranin (Fig. 6c), similar to the findings of the lung lesion under bronchoscopy (Fig. 6d, e, f). Based on these findings, amrubicin was changed to nogitecan as fourth-line chemotherapy. The patient continues chemotherapy and is still alive.

\section{Discussion}

Most patients with gastrointestinal metastases have no specific symptoms. Thus, although 4.7-14\% [1,6] of autopsy cases with lung cancer showed gastrointestinal metastases, the prevalence of gastrointestinal metastases from lung cancer that were found during the patients' lifetime was much lower, ranging from $0.5 \%$ to $1.77 \%[7,8]$. Symptomatic small intestinal metastases have rarely been reported $[3,7,9]$. Physicians do not usually suspect gastrointestinal metastases unless the patient has symptoms. Clinical symptoms of gastrointestinal metastases from lung cancer vary, including gastrointestinal perforation, obstruction, and hemorrhage. Including the present case, a total of six cases diagnosed as small bowel metastases from small cell lung cancer during the patients' lifetime have been reported (Table 1) [3, 8, 10-12]. All of these, except the present case, required surgery. It was possible in the present case to diagnose asymptomatic small intestinal metastasis from small cell lung cancer by PET and EUS-FNA; to the best of our knowledge, this is the first such report.

All types of lung cancer may disseminate to any segment of the small bowel. In one series, squamous cell carcinoma was the most common cell type, followed by large cell carcinoma, small cell carcinoma, and adenocarcinoma [6]. Squamous cell carcinoma was also the most frequent histological type in other reported series [7, 9]. Most small intestinal metastases are found in the jejunum, followed by the duodenum $[1,13]$. In 

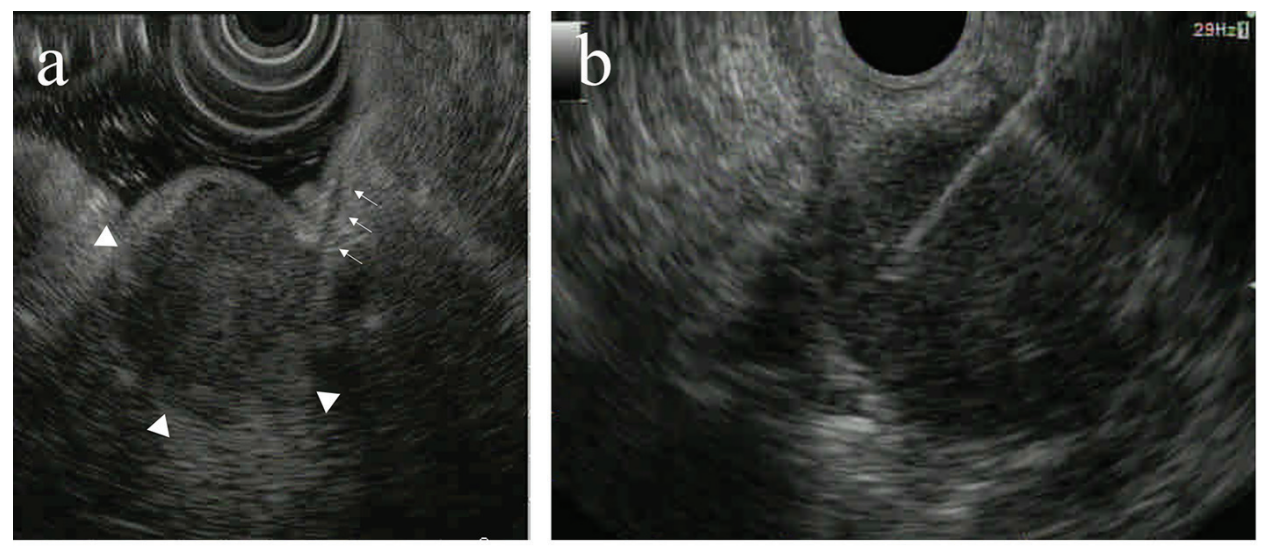

Figure 4. The submucosal tumor (SMT) was visualized as $30 \mathrm{~mm}$ in size, round-shaped, homogeneous, and low echoic mass (arrowheads) connected with fourth layer of duodenal wall (arrows) on radial type endoscopic ultrasound (EUS) (a). EUS-guided fine-needle aspiration (FNA) was performed for the SMT (b).

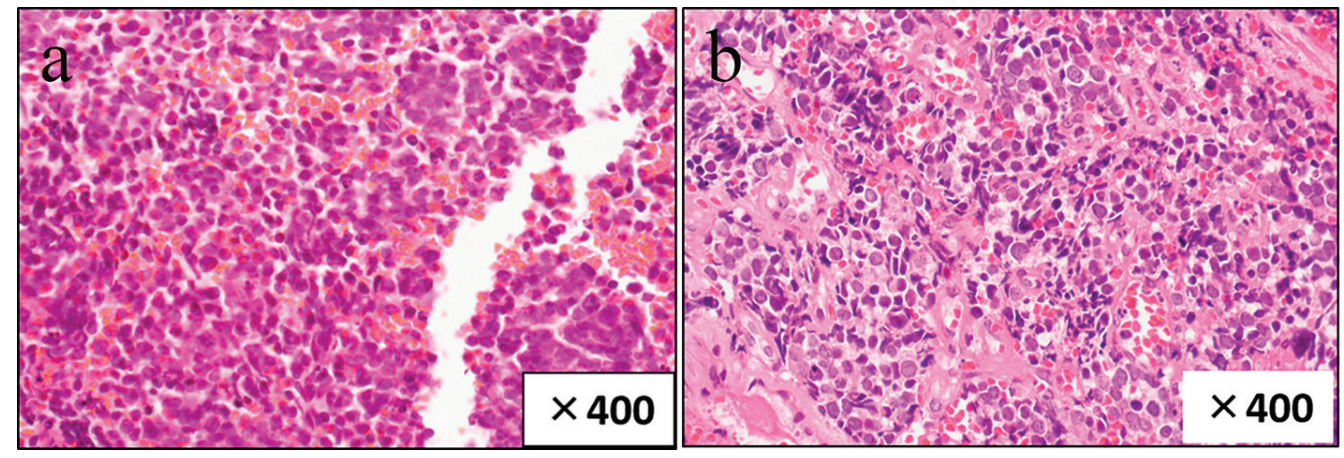

Figure 5. HE (hematoxylin and eosin)-stained sections of specimens obtained by endoscopic ultrasound-guided fine-needle aspiration (EUS-FNA) show relatively small atypical cells with a high nuclear/cytoplasm ratio (a), similar to those of the primary lung lesion (b).
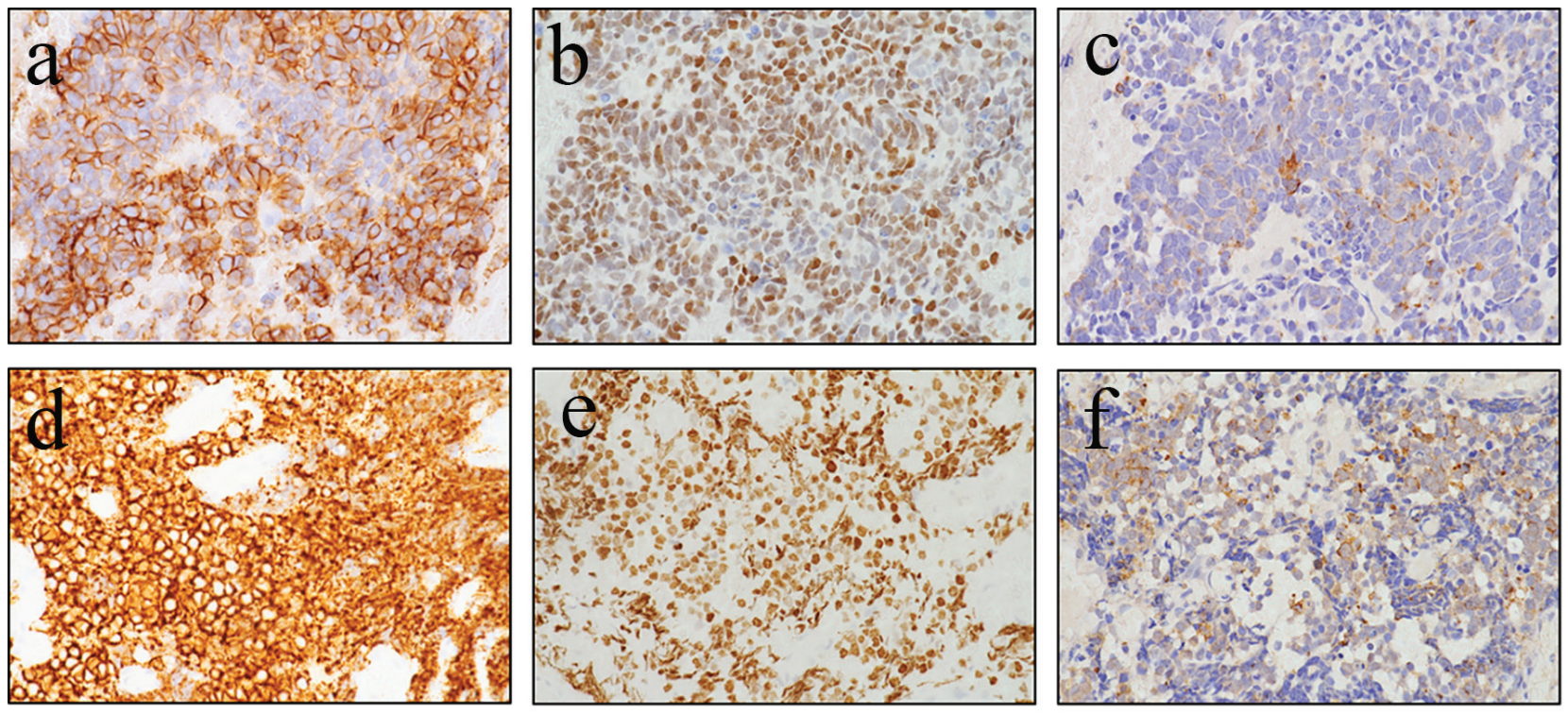

Figure 6. Tumor cells are diffusely positive for cluster of differentiation 56 (CD56) (a) and thyroid transcription factor 1 (TTF-1) (b) and focally positive for chromogranin (c), similar to those obtained from the primary lung lesion under bronchoscopy (d: CD56; e: TTF-1; f: chromogranin). 


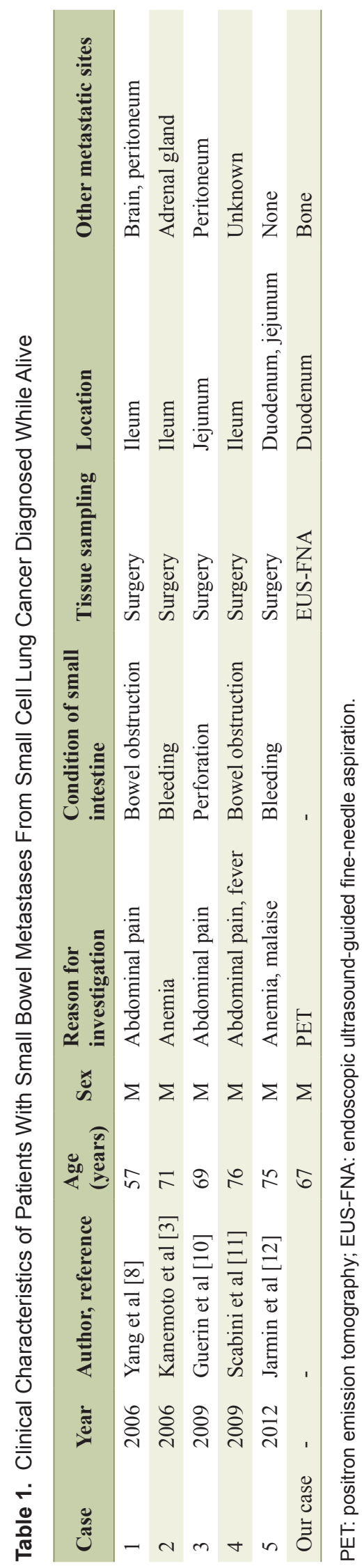

contrast, Berger et al reported six small intestinal metastases in two patients, in the ileum in three, and in both sites in two [7].

The clinical usefulness of PET scanning has been reported in gastrointestinal carcinoma, such as preoperative staging of esophageal cancer and recurrent colorectal carcinoma [14]. Kelly et al evaluated the additional effect of FDG-PET on staging accuracy and cost-effectiveness compared to CT scanning alone on the preoperative staging of non-small cell lung cancer [15]. Shiono et al reported a case in which PET detected small intestinal metastasis from lung cancer [16]. In the present case, PET contributed to the discovery of asymptomatic gastrointestinal metastasis. Although there is no consensus on the role of FDG-PET in the diagnosis of metastasis from lung cancer, PET may become a useful option to assess gastrointestinal metastases from lung cancer.

To obtain specimens from SMT via biopsy under endoscopy is frequently difficult, because the mucosal surface of SMT is usually normal. EUS is useful to diagnose these lesions [17]. EUS-FNA is considered to be a more useful technique for the diagnosis of SMT in the gastrointestinal tract [18]. The collection rate of sufficient specimens from an SMT of the gastrointestinal tract by EUS-FNA was reported to be $74.5-91.8 \%$ in past studies $[18,19]$. In the present case, EUS-FNA was sufficient to gain adequate material for immunohistological diagnosis, which was essential for diagnosing metastasis from lung cancer. Because EUS-FNA can diagnose not only SMT of the gastrointestinal tract but also lesions in many other organs, such as the adrenal glands or lymph nodes [4], the procedure could become a powerful tool to diagnose metastases from lung cancer.

A case of asymptomatic duodenal metastasis from small cell lung cancer was described. PET was the first investigation to indicate the gastrointestinal metastasis. IHC for specimens obtained by EUS-FNA was useful to confirm the diagnosis. This information contributed to the subsequent treatment strategy.

\section{Competing Interests}

There are no financial relationships to disclose for any of the authors.

\section{Abbreviations}

EUS-FNA: endoscopic ultrasound-guided fine-needle aspiration; IHC: immunohistochemistry; PET: positron emission tomography; FDG: fluorodeoxyglucose; CT: computed tomography; CEA: carcinoembryonic antigen; NSE: neuron-specific enolase; SCC: squamous cell carcinoma; SLX: sialyl Lewis X-i; CYFRA: cytokeratin 19 fragment; ProGRP: pro gastrinreleasing peptide; SMT: submucosal tumor; HE: hematoxylin and eosin; GIST: gastrointestinal stromal tumor; CD: cluster of differentiation; TTF-1: thyroid transcription factor 1.

\section{References}

1. Yoshimoto A, Kasahara K, Kawashima A. Gastrointesti- 
nal metastases from primary lung cancer. Eur J Cancer. 2006;42(18):3157-3160.

2. Liu W, Zhou W, Qi WL, Ma YD, Xu YY. Gastrointestinal hemorrhage due to ileal metastasis from primary lung cancer. World J Gastroenterol. 2015;21(11):34353440 .

3. Kanemoto K, Kurishima K, Ishikawa H, Shiotani S, Satoh H, Ohtsuka M. Small intestinal metastasis from small cell lung cancer. Intern Med. 2006;45(16):967-970.

4. Yamao K, Sawaki A, Mizuno N, Shimizu Y, Yatabe Y, Koshikawa T. Endoscopic ultrasound-guided fine-needle aspiration biopsy (EUS-FNAB): past, present, and future. J Gastroenterol. 2005;40(11):1013-1023.

5. The Japan Lung Cancer Society. General rules for clinical and pathological recording of lung cancer. 7 th ed. Tokyo: Kanehara \& Co., Ltd.; 2010.

6. Antler AS, Ough Y, Pitchumoni CS, Davidian M, Thelmo W. Gastrointestinal metastases from malignant tumors of the lung. Cancer. 1982;49(1):170-172.

7. Berger A, Cellier C, Daniel C, Kron C, Riquet M, Barbier JP, Cugnenc PH, et al. Small bowel metastases from primary carcinoma of the lung: clinical findings and outcome. Am J Gastroenterol. 1999;94(7):1884-1887.

8. Yang CJ, Hwang JJ, Kang WY, Chong IW, Wang TH, Sheu CC, Tsai JR, et al. Gastro-intestinal metastasis of primary lung carcinoma: clinical presentations and outcome. Lung Cancer. 2006;54(3):319-323.

9. McNeill PM, Wagman LD, Neifeld JP. Small bowel metastases from primary carcinoma of the lung. Cancer. 1987;59(8):1486-1489.

10. Guerin E, Gilbert O, Dequanter D. Acute abdomen: a rare presentation of lung cancer metastasis. Case Rep Med. 2009;2009:903897.

11. Scabini S, Rimini E, Romairone E, Scordamaglia R, Bog- gio M, Musizzano Y, Ferrando V. Small bowel metastasis from primary neuroendocrine small cell lung carcinoma. Chir Ital. 2009;61(5-6):679-682.

12. Jarmin R, Azman A, Rahim R, Kosai NR, Das S. A rare case of intussusception associated with metastasized small cell carcinoma of lung. Acta Med Iran. 2012;50(11):782784.

13. Jansen JM, Oldenburg B, van Milligen de Wit AW. Small intestinal metastasis from non-small-cell carcinoma of the lung: a rare cause of GI bleeding of obscure origin. Gastrointest Endosc. 2004;59(3):447-449.

14. Hustinx R. PET imaging in assessing gastrointestinal tumors. Radiol Clin North Am. 2004;42(6):1123-1139, ix.

15. Kelly RF, Tran T, Holmstrom A, Murar J, Segurola RJ, Jr. Accuracy and cost-effectiveness of [18F]-2-fluorodeoxy-D-glucose-positron emission tomography scan in potentially resectable non-small cell lung cancer. Chest. 2004;125(4):1413-1423.

16. Shiono S, Masaoka T, Sato T, Yanagawa N. [Positron emission tomography (PET)-computed tomography (CT) suggesting small intestinal metastasis from lung cancer; report of a case]. Kyobu Geka. 2006;59(5):426-429.

17. Byrne MF, Jowell PS. Gastrointestinal imaging: endoscopic ultrasound. Gastroenterology. 2002;122(6):16311648.

18. Ando N, Goto H, Niwa Y, Hirooka Y, Ohmiya N, Nagasaka T, Hayakawa T. The diagnosis of GI stromal tumors with EUS-guided fine needle aspiration with immunohistochemical analysis. Gastrointest Endosc. 2002;55(1):3743.

19. Suzuki T, Arai M, Matsumura T, Arai E, Hata S, Maruoka D, Tanaka T, et al. Factors Associated with Inadequate Tissue Yield in EUS-FNA for Gastric SMT. ISRN Gastroenterol. 2011;2011:619128. 